\title{
Physical Activity and Nutrition in Primary and Tertiary Prevention of Colorectal Cancer
}

\author{
Michael H. Schoenberg \\ Medical Center for Optimized Cancer Therapy, Munich, Germany
}

\section{Keywords}

Colorectal cancer - Physical activity - Mediterranean diet . Prognosis - Quality of life

\section{Summary}

Background: Lifestyle factors play a pivotal role in the primary and tertiary prevention of colorectal cancer. The purpose of this review article is to summarize data concerning the effect of the lifestyle factors physical activity (PA) and nutrition in primary and, more importantly, tertiary prevention of colorectal cancer (CRC). Methods: Focusing on the influence of lifestyle factors on prognosis und quality of life (OOL), a comprehensive literature search of clinical studies published mainly in the years 2000 until 2015 was performed and the current knowledge based on these clinical studies reviewed. Results: Besides avoiding risk factors (such as smoking and overindulgence in alcohol), healthy weight, regular and moderate PA as well as a diet which contains fruit, vegetables, poultry, and fish (so-called 'Mediterranean' diet) may reduce the risk of the disease significantly. Patients already diagnosed with $\mathrm{CRC}$ can also actively improve the prognosis of $\mathrm{CRC}$ and $\mathrm{QOL}$ by changing their lifestyle. Patients commencing moderate exercise and modifying their eating habits in terms of a 'Mediterranean' diet can reduce cancer-specific and overall mortality by up to $40 \%$ and significantly increase their quality of life already during chemotherapy. Therefore, moderate physical exercise, calorie restriction, and a Mediterranean dietary pattern for patients with $\mathrm{CRC}$ should be recommended by physicians treating these patients. In fact, the World Cancer Research Fund/American Institute for Cancer Research (AICR/WCRF) systematic literature review from 2007 shows that the lifestyle changes recommended after diagnosis are the same for primary prevention of this disease. Conclusion: Lifestyle changes such as moderate PA and a Mediterranean diet significantly improve the $\mathrm{QOL}$ as well as the prognosis of patients suffering from colorectal disease. However, the effect of lifestyle changes is mostly based on observational studies, while only few studies are prospective and none are randomized. Therefore, these observational studies warrant controlled randomized trials to prove the effectiveness of lifestyle interventions on QOL and cancer recurrence.

(c) 2016 S. Karger GmbH, Freiburg

\section{Colorectal Cancer and Physical Activity}

Primary Prevention

The worldwide incidence of colorectal cancer (CRC) varies to a large extent. In underdeveloped countries the incidence amounts to $1-5$ patients per 100,000 inhabitants while it can increase to 20-60 patients per 100,000 inhabitants in industrialized regions. It is well established that besides genetic dispositions, lifestyle factors such as too little physical activity (PA), alcohol consumption, nutrition, and smoking are relevant risk factors [1]. These effects are not only relevant regarding primary prevention but also in tertiary prevention.

Regarding primary prevention, convincing evidence shows that $\mathrm{PA}$, either recreational or during work, may prevent the development of CRC.

Wolin et al. [2] showed in a meta-analysis including 52 observational and cohort studies that recreational PA could significantly reduce the incidence of colon cancer by altogether $24 \%$. Furthermore, in an earlier epidemiological study of 150,000 participants with a follow-up of 6 years, it was found that $4-6 \mathrm{~h}$ of exercise per

\begin{tabular}{|c|c|}
\hline KARGER & $\begin{array}{l}\text { (c) } 2016 \text { S. Karger GmbH, Freiburg } \\
2297-4725 / 16 / 0323-0199 \$ 39.50 / 0\end{array}$ \\
\hline Fax +497614520714 & \\
\hline $\begin{array}{l}\text { Information@Karger.com } \\
\text { www.karger.com }\end{array}$ & $\begin{array}{l}\text { Accessible online at: } \\
\text { www.karger.com/vis }\end{array}$ \\
\hline
\end{tabular}


week could possibly reduce the risk of colon and rectal cancer by 13 and 30\%, respectively [3]. Thus, the risk of colon cancer significantly decreased with increasing total hours of recreational sports, whereas this effect was not seen in rectal cancer.

A recent European study showed similar results. In this large cohort of 350,000 participants observed over a period of 12 years, it was shown that a healthy lifestyle such as normal weight, no smoking, little alcohol intake, and moderate PA could clearly decrease the frequency of CRC up to $37 \%$ [4]. This association was seen in colon as well as rectal cancer. Sufficient PA alone decreased the likelihood of colon cancer by $13 \%$. This effect was not observed for rectal cancer.

The authors concluded that overall $16 \%$ of the CRC cases which were diagnosed during the observation period were attributable to the fact that the patients did not follow the various lifestyle factors recommended.

These data show a general inverse relation of PA and the incidence of CRC. In the studies, however, a distinction between the tumor sites was not drawn. A recently published meta-analysis, additionally examining the subsites of the colon, could show that the indirect correlation between colon cancer risk and exercise did not differ between the colonic subsites (i.e. right vs. left hemicolon) [5]

In contrast, the risk reduction for rectal cancer by PA still remains doubtful. In two earlier studies a positive effect of PA on reducing the risk of rectal cancer could be seen $[6,7]$. Shortly thereafter, Steindorf et al. [8] could not reproduce these positive results. In this study, rectal cancer risk was not inversely associated with PA. Apparently, this topic warrants further research and might indicate different mechanisms leading to the development of either colon or rectal cancer.

\section{Tertiary Prevention (Physical Activity as an Element of Best Supportive Care during Cancer Treatment)}

The purpose of this review, however, is to focus on the effect of PA after diagnosis of CRC (tertiary prevention) and its impact on quality of life (QOL) and prognosis. The prognosis of the disease will thereby be defined as overall and disease-free survival.

Diagnosis and treatment of CRC, in most cases including operation, chemotherapy, and in some instances radiation, naturally leads to an increasing inactivity of the patient. The side effects are, amongst others, anemia, leukopenia, nausea, diarrhea, and vomiting as well as, depending on the substances used, cardiomyopathy. This evidently impairs the functional PA of the patients. Moreover, patients often suffer from cancer-related fatigue leading to weariness, weakness, and in part to depression. These both physical and mental symptoms during therapy may decrease the ability to commence with and maintain sufficient PA.

Courneya et al. [9] and Dimeo et al. [10] showed in earlier studies with lymphoma and breast cancer patients that PA during primary therapy is beneficial and counteracts the side effects of the oncological treatment. Patients who are or remain active during this period do not suffer to such an extent from typical side effects such as fatigue etc., and adhere to the (chemo-)therapy at a higher percentage rate. In the 'Physical Activity during Cancer Treatment' (PACT) trial recently conducted in the Netherlands, colon cancer patients undergoing chemotherapy were randomly assigned to either a group receiving a supervised exercise program or to 'usual care' without intervention. The patients in the exercise group experienced significantly less physical fatigue and reported higher levels of physical functioning compared to patients in the 'usual care' group [11]. Therefore, adding a program of moderate PA to best supportive care during this phase could significantly improve QOL and contribute to the wellbeing of the patients.

Recently emerging observational data have shown that CRC survivors who are physically active also reduce their risk of cancer recurrence and overall mortality. In the meantime, seven prospective studies have supported this notion.

A study conducted in Australia observed 41,528 patients with CRC for 5.5 years. Although all patients were treated by the same oncological standards, the physically active patients had a significantly better prognosis. The overall mortality of the active patients was $23 \%$ lower than in the inactive group [12]. Being physically active was defined as training for at least 20 min several times a week.

Interestingly, these positive results were only seen in patients with colon cancer stages II and III based on the American Joint Committee on Cancer (AJCC) staging system. In these relatively frequent stages of the disease the improvement of the overall and cancer-specific survival amounted to $39 \%$ and even to $51 \%$, respectively. PA in earlier (AJCC I) and later stages (AJCC IV) had no impact on the prognosis. This study, however, did not differentiate between colon and rectal cancer.

In the same year a prospective study with colon cancer patients stage III according to the AJCC could show that moderate training was sufficient to improve the prognosis by up to $45 \%$.

PA was assessed by the metabolic equivalent task per hour (MET-h) score as extensively described for every possible PA by Ainsworth et al. [13, 14]. Meyerhardt et al. [15] found that 18 MET-h accumulated per week had the best effects concerning QOL and prognosis (fig. 1).

In a subsequent study the researchers looked only at women with colon cancer (stage I-III) and found that physically active female patients improved their overall mortality by $51 \%$ [16]. Counterintuitively, formerly inactive patients had the most pronounced benefit from exercise as compared to those who were active before. Thus, it seems to be essential to begin with PA immediately after diagnosis, regardless of how active one was before the disease. In a further study by Meyerhardt et al. [17], the authors found that the effect of PA on prognosis seemed to be even 'dose-dependent'. In men with non-metastatic CRC, increased PA was associated with a lower risk of CRC-specific and overall mortality. In fact, male patients whose activity exceeded 27 MET-h per week improved their prognosis by over $50 \%$. This benefit of PA was detectable regardless of age, stage, body mass index (BMI), year of diagnosis, location of tumor, and pre-diagnosis PA [17].

These results were reproduced by several studies internationally. In the 'Active Australian Survey', Baade et al. [18] differenti- 


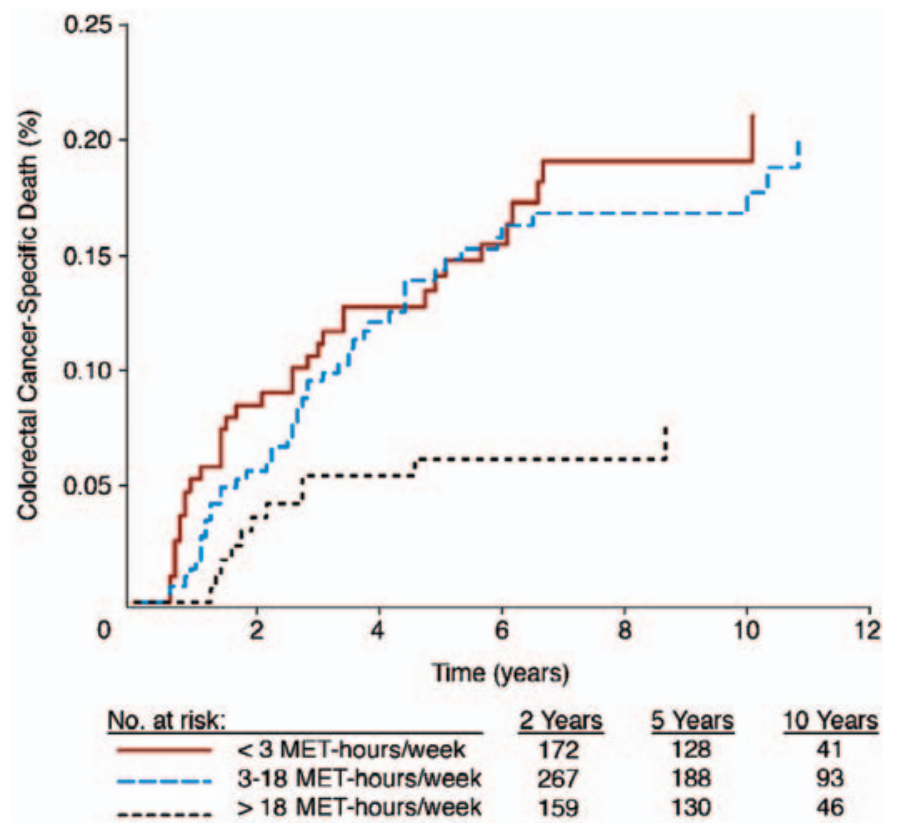

Fig. 1. Effect of physical activity by MET-h per week and disease-specific mortality in \% (reprinted with permission from [16]. ${ }^{\circ}$ American Society of Clinical Oncology. All rights reserved).

ated three groups of patients with CRC without differentiating between colon and rectal cancer. The first group consisted of patients who were completely inactive. The second group did not meet the recommendations of the American College of Sports Medicine (150 min of moderate PA per week) whereas the third group met these recommendations. These results supported the observations made by Meyerhardt et al. [15-17], although the improvement of the prognosis in the sufficiently active third group amounted only to $25 \%$. If the patients kept up and even increased their PA as assessed 12 months after diagnosis, the positive effect reached over $30 \%$ [18].

Recently, Kuiper et al. [19] published the results of a study conducted in the Netherlands in which 1,339 women were followed up for 12 years. Patients who were active before diagnosis clearly experienced an improvement of the CRC-specific mortality by $32 \%$ and of the all-cause mortality by $37 \%$. Interestingly, the benefit in prognosis was seen if formerly inactive patients started to train sufficiently after diagnosis. The message of this study was to recommend patients to get started regardless of their activity level before falling ill [19].

Additionally, Campbell et al. [20] looked at patients from the opposite perspective. 2,300 patients were followed up for over 16 years. The authors of this study could show that patients who were sitting more than $6 \mathrm{~h} /$ day post diagnosis worsened the prognosis of the disease (all-cause mortality) by $27 \%$. The cancer-related mortality even increased up to $63 \%$ in this study [20].

The results of these studies led to the notion that sedentary lifestyle and tobacco smoking are equally dangerous after cancer diagnosis and coined the statement 'it is never too early and very seldom too late to start an active life'.

Physical Activity and Nutrition in Prevention of CRC
These results in CRC patients are in line with observations seen in other malignant diseases (breast, prostate, pancreas, and lung cancer).

In a meta-analysis of various studies which included 49,095 cancer survivors, the investigators could show that breast or colorectal cancer survivors who increased their PA by any level from pre- to post-diagnosis showed a total mortality risk decreased by $39 \%$ compared with those who did not change their PA level or who were inactive/insufficiently active before diagnosis [21].

In this analysis, every increase of PA by 10 MET-h per week led to a mortality rate reduction of $28 \%$; thus, as already mentioned, this effect seemed to be dose-dependent but is naturally limited [21].

Moreover, a randomized study of exercise intervention in colorectal patients showed that the patients who were able to increase their aerobic fitness by either intervention or information also improved not only their prognosis but also their QOL, physical functioning, and psychosocial stress significantly, as compared to patients who did not exhibit the same positive development of their aerobic fitness [22].

These data have led to the initiation of the randomized controlled 'Colon Health and Life-Long Exercise Change' (Challenge) trial. The goal is to determine the effects of a 3-year structured PA intervention in patients suffering from colon cancer stage UICC II and UICC III. To date, this study is ongoing, and it will be interesting to see the final results.

\section{Pathophysiology}

The physiological pathways by which PA improves QOL and prognosis are still not clear. Moderate PA has most likely several physiological effects which might also interact and change the course of CRC.

One mechanism proposed is insulin as well as the effect of different insulin growth factors on carcinogenesis. Enhanced insulin metabolism prevents apoptosis and leads to uncontrolled cell growth. This theory is substantiated by the fact that patients who are obese and suffer from insulin resistance exhibit an increased risk for colon cancer [23]. PA obviously decreases the levels of insulin and reduces insulin resistance, thus counteracting the carcinogenic insulin axis (for review see [24]).

Another risk factor for several different cancers including CRC is chronic inflammation and an impaired immune system. However, the mechanisms underlying the contribution of inflammation to cancer remain elusive. Proinflammatory mediators such as cyclooxygenase 2 (COX-2) and prostaglandin E2 (PGE2) contribute to cancer. Recently it could be shown that COX-2 is induced by proinflammatory cytokines and that its concentrations are elevated in human colon cancer cells. Furthermore, COX-2-derived PGE2 promotes colonic tumor growth via silencing certain tumor suppressors and DNA repair genes by DNA methylation in colon tumor cells.

Moderate PA is able to stabilize the immune system, thus preventing proliferation of tumor cells. Moreover, Demarzo et al. [25] 
found in an animal experiment that exercise training had remarkable antiproliferative and anti-inflammatory effects in the colon mucosa of the rat, consequently reducing also colon cancer cell proliferation in rats.

The authors suggested that this may be an important mechanism of how exercise protects against colon cancer. These findings do not only support a rationale to treat these proinflammatory pathways for cancer prevention but also provide a new approach to the development of a new therapy for subverting chronic inflammation- and tumor-induced immunosuppression.

Multiple epidemiologic studies, randomized clinical trials in patients, i.e. with hereditary CRC, and pooled analyses of patients who were taking non-steroidal anti-inflammatory drugs (NSAIDs) for the prevention of cardiovascular disease could show that conventional NSAIDs are able to prevent CRC to a certain extent. However, chronic medication with NSAIDs also bears various serious side effects. Hopefully, newer and chemically modified NSAIDs could be more effective and safe in the future. Moreover, recent work also indicates that targeting earlier stages of colorectal carcinogenesis with these new NSAIDs could be a promising approach for treating this malignant disease, since it may only require a relatively short use of these therapeutics [26].

In line with this notion and with the effect of PA on proinflammatory mediators such as PGE2, Yamauchi et al. [27] utilized the molecular pathological database of stage I-III colon and rectal cancer derived from two previously performed prospective cohort studies (i.e. the Nurse's Health Study and the Health Professionals Follow-up Study). The authors determined patient survival according to the PA of the patients after diagnosis and correlated these data with the PTGS2 (the official symbol for COX-2) status in the tumor tissue (with 382 PTGS2-positive and 223 PTGS2-negative tumors by means of immunohistochemistry). PTGS2 and its enzymatic product PGE2 are, as already mentioned, key contributors to inflammatory responses and play important roles in CRC development and progression. Post-diagnosis PA was only associated with better survival among patients if their tumor was PTGS2-positive, but not with PTGS2-negative tumors. These findings do not only elucidate new insights in the biology of CRC, adding to the expanding literature on the effects of proinflammatory mediators on tumor growth, but might also influence clinical recommendations concerning the efficacy of lifestyle changes after a diagnosis of CRC.

Another physiological pathway explaining the positive effects of PA are the enhanced levels of myokines, which are proteins produced and secreted by muscle cells in response to exercise. Myokines have been shown to induce multiple health benefits including improved metabolic and anti-inflammatory effects in various organs.

In a mouse model, Aoi et al. [28] reported that regular exercise prevents the formation of aberrant crypt foci, which are the precursor lesions of adenocarcinoma on the mucosal surface of colon tissue. Recently the same authors identified a new myokine, i.e. secreted protein acidic and rich in cysteine (SPARC), which is secreted from skeletal muscle into the circulation in response to exercise in both mice and humans. In a colon cancer mouse model, regular moderate exercise significantly reduced the formation of aberrant crypt foci in wild-type mice. Surprisingly, this effect of moderate PA was not observed in SPARC-null mice. The authors concluded from this animal experiment that SPARC myokines possibly prevent the formation of an early stage of colon cancer. They hypothesized that an administration of SPARC-like proteins may be useful for the prevention and treatment of colon cancer [28].

Little evidence is available concerning the relation of PA with colon adenomas, a colon cancer precursor. Wolin et al. [29] identified a total of 20 studies investigating the effect of PA and the risk or prevalence of colon adenoma or polyps. The authors could show a significant inverse association between PA and colon adenomas both in men and women. The association appeared to be even more pronounced concerning larger or more advanced polyps. This meta-analysis confirming previous reports of an inverse association of PA and colon adenoma could be an additional explanation for the effect of PA against colon cancer [29].

\section{Nutrition and Colon Cancer}

It is well established that obesity is a risk factor for the development of various cancers including breast, esophageal, endometrial, kidney, and also colon cancer. The influence of the two important parameters 'height' and 'weight' as well as the calculated BMI was studied in a prospective, observational study of patients who had stage III colon cancer [30]. In the beginning, 35\% of patients were overweight (BMI $25-29.9 \mathrm{~kg} / \mathrm{m}^{2}$ ), and $34 \%$ were obese (BMI $>30$ $\mathrm{kg} / \mathrm{m}^{2}$ ). Surprisingly, an increased BMI (obesity: BMI $>35 \mathrm{~kg} / \mathrm{m}^{2}$ ) was not associated with a higher risk of colon cancer recurrence or death. Furthermore, BMI and weight changes (lower or higher) during the observation period did not influence cancer recurrence rate and/or mortality [30]. The authors concluded that neither BMI nor weight change were associated with the prognosis of colon cancer.

Recently, Campbell et al. [31] analyzed the data of the Cancer Prevention Study-II Nutrition Cohort including 184,000 participants and evaluated the association of BMI before and after diagnosis of non-metastatic CRC with survival in this prospective cohort. The authors found that an increased BMI before CRC diagnosis was associated with a higher risk of mortality resulting from all causes, related to CRC or cardiovascular diseases. Interestingly, post diagnosis BMI (based on weight reported) was not associated with all-cause or cancer-specific mortality. The authors concluded that the BMI before but not after diagnosis was an important predictor of survival among patients with non-metastatic CRC [31].

Nevertheless, since obesity plays an important role in the prognosis and the course of disease, it is logical to study different diets as a risk factor. In a prospective study, Meyerhardt et al. [32] correlated the dietary patterns of patients with stage III colon cancer with cancer recurrences and mortality. They identified two eating habits which were characterized by either high intake of meat, fat, refined grains, and dessert (Western diet) or a diet containing fruit, vegetables, poultry, and fish (so-called 'Mediterranean' diet). 
The authors found (median follow-up: 5.3 years) that a Western diet, which features an especially high intake of red meat, was associated with a three times higher risk of colon cancer recurrences or death. Interestingly, the worse prognosis induced by Western diet was not significantly modified by sex, age, nodal stage, and BMI. In a recent study, the same research group looked more closely at diet elements and the influence of the so-called glycemic load on survival among colon cancer patients. In this study, patients suffering from stage III colon cancer reported on their dietary intake of carbohydrates for 6 months. The authors could show that an increased glycemic load was unfavorable for the prognosis of the disease. However, this inverse relationship depended on the BMI. In patients with a BMI $<25 \mathrm{~kg} / \mathrm{m}^{2}$ an increased glycemic load had no negative effect on the disease-free survival, whereas in obese patients the glycemic load was statistically significant associated with a worse disease-free survival. Similarly, increased total carbohydrate intake was also associated with inferior disease-free, recurrence-free, and overall survival. Therefore, restriction of carbohydrates and dietary glycemic load might improve the patients' survival, especially if they are obese. Moreover, in the post-diagnosis period it seems to be essential to optimize the body weight to a normal range [32].

Dietary supplements such as vitamins and minerals have been studied extensively over the past 30 years. In fact, approximately $60-80 \%$ of cancer survivors consume these supplements regularly. Low vitamin D levels have been shown to increase the risk of developing colon cancer, and $\mathrm{Ng}$ et al. [33] could show that patients with CRC who exhibited normal or higher plasma vitamin D levels before diagnosis had a better overall survival. The effect of vitamin $\mathrm{D}$ on the prognosis remains unknown. Nevertheless, these results suggest that in patients with low vitamin D levels a careful substitution to normal levels could be beneficial.

Because colon cancer often emerges from adenomatous polyps, prevention of polyp recurrences is expected to be beneficial. Except for the effects of PA, other clinical trials with antioxidants, fiberrich supplements, increased fruit and vegetable intake, or folate have failed to show any positive effect on preventing the new formation of polyps. In fact, it actually seems to encourage the growth of tumor cells once they are present $[34,35]$.

\section{Conclusion}

In the last two decades, the treatment of colon as well as rectal cancer has been rather successful. Nowadays, studies with good quality show a 5 -year disease-free survival of $82.7 \%$ for stage II colon cancer and a 5-year disease-free survival in stage III with adjuvant chemotherapy of $63.8 \%$ [36]. In stage I, the 5-year diseasefree survival is greater than $90 \%$, while it is slightly higher than $10 \%$ in patients with stage IV disease [37]. In rectal cancer, qualityapproved total mesorectal excision (TME) surgery had a major impact on cancer outcomes, showing the importance of good surgical technique and teaching [38]. In combination with preoperative radiochemotherapy followed by TME surgery, this strategy has decreased the 5- and 10-year local recurrence rates below 10\%. However, the problem of distant liver metastases (35-45\%) is still unresolved [39]. Besides these improvements, lifestyle factors play a pivotal role in the development of CRC. Regular and moderate PA as well as a Mediterranean diet may reduce the risk of the disease significantly. Even more patients already diagnosed with CRC can actively improve their prognosis by changing their lifestyle, i.e. stop drinking alcohol and smoking, trying to optimize their weight, starting regular moderate exercise, and changing their eating habits with meals mainly containing fruit, vegetables, poultry, and fish (so-called 'Mediterranean' diet). A recent study showed that a pescovegetarian diet (vegetarians who also consume fish and seafood) reduced the risk by $43 \%$ [40].

Nevertheless, lifestyle intervention trials are needed to substantiate these observational results regarding cancer recurrences. However, even now there is no contraindication for promoting moderate physical exercise, moderate calorie restriction, and a Mediterranean dietary pattern. In fact, the AICR/WCRF systematic literature review from 2007 recommends cancer patients suffering from CRC to adopt this lifestyle recommended for the prevention of this disease [41].

\section{Disclosure Statement}

The author declares that there is no conflict of interest.

\section{References}

1 Washington MK: Colorectal carcinoma: selected issues in pathologic examination and staging and determination of prognostic factors. Arch Pathol Lab Med 2008; 132:1600-1607.

2 Wolin KY, Yan Y, Colditz GA, Lee IM: Physical activity and colon cancer prevention: a meta-analysis. $\mathrm{Br} \mathrm{J}$ Cancer 2009;100:611-616.

3 Chao A, Connell CJ, Jacobs EJ, McCullough ML, Patel AV, Calle EE, Cokkinides VE, Thun MJ: Amount, type, and timing of recreational physical activity in relation to colon and rectal cancer in older adults: the Cancer Prevention Study II Nutrition Cohort. Cancer Epidemiol Biomarkers Prev 2004;13:2187-2195.
4 Aleksandrova K, Pischon T, Jenab M, et al: Combined impact of healthy lifestyle factors on colorectal cancer: a large European cohort study. BMC Med 2014;12:168.

5 Boyle T, Keegel T, Bull F, Heyworth J, Fritschi L: Physical activity and risks of proximal and distal colon cancers: a systematic review and meta-analysis. J Natl Cancer Inst 2012;104:1548-1561.

6 Colbert LH, Hartman TJ, Malila N, Limburg PJ, Pietinen P, Virtamo J, Taylor PR, Albanes D: Physical activity in relation to cancer of the colon and rectum in a cohort of male smokers. Cancer Epidemiol Biomarkers Prev 2001;10:265-268.
Slattery ML, Edwards S, Curtin K, Ma K, Edwards R, Holubkov R, Schaffer D: Physical activity and colorectal cancer. Am J Epidemiol 2003;158:214-224.

8 Steindorf K, Jedrychowski W, Schmidt M, Popiela T Penar A, Galas A, Wahrendorf J: Case-control study of lifetime occupational and recreational physical activity and risks of colon and rectal cancer. Eur J Cancer Prev 2005; 14:363-371.

9 Courneya KS, Segal RJ, Mackey JR, et al: Effects of aerobic and resistance exercise in breast cancer patients receiving adjuvant chemotherapy: a multicenter randomized controlled trial. J Clin Oncol 2007;25:43964404 . 
10 Dimeo FC, Stieglitz RD, Novelli-Fischer U, Fetscher S, Keul J: Effects of physical activity on the fatigue and psychologic status of cancer patients during chemotherapy. Cancer 1999;85:2273-2277.

11 van Vulpen JK, Velthuis MJ, Steins Bisschop CN, et al: Effects of an exercise program in colon cancer patients undergoing chemotherapy. Med Sci Sports Exerc 2015. 48:767-775.

12 Haydon AM, Macinnis RJ, English DR, Giles GG: Effect of physical activity and body size on survival after diagnosis with colorectal cancer. Gut 2006;55:62-67.

13 Ainsworth BE, Haskell WL, Herrmann SD, Meckes N, Bassett DR Jr, Tudor-Locke C, Greer JL, Vezina J, Whitt-Glover MC, Leon AS: 2011 Compendium of Physical Activities: a second update of codes and MET values. Med Sci Sports Exerc 2011;43:1575-1581.

14 Ainsworth BE, Haskell WL, Whitt MC, et al: Compendium of physical activities: an update of activity codes and MET intensities. Med Sci Sports Exerc 2000;32: S498-504.

15 Meyerhardt JA, Heseltine D, Niedzwiecki D, et al: Impact of physical activity on cancer recurrence and survival in patients with stage III colon cancer: findings from CALGB 89803. J Clin Oncol 2006;24:3535-3541.

16 Meyerhardt JA, Giovannucci EL, Holmes MD, Chan AT, Chan JA, Colditz GA, Fuchs CS: Physical activity and survival after colorectal cancer diagnosis. J Clin Oncol 2006;24:3527-3534.

17 Meyerhardt JA, Giovannucci EL, Ogino S, Kirkner GJ, Chan AT, Willett W, Fuchs CS: Physical activity and male colorectal cancer survival. Arch Intern Med 2009; 169:2102-2108.

18 Baade PD, Meng X, Youl PH, Aitken JF, Dunn J, Chambers SK: The impact of body mass index and physical activity on mortality among patients with colorectal cancer in Queensland, Australia. Cancer Epidemiol Biomarkers Prev 2011;20:1410-1420.

19 Kuiper JG, Phipps AI, Neuhouser ML, et al: Recreational physical activity, body mass index, and survival in women with colorectal cancer. Cancer Causes Control 2012;23:1939-1948.

20 Campbell PT, Patel AV, Newton CC, Jacobs EJ, Gapstur SM: Associations of recreational physical activity and leisure time spent sitting with colorectal cancer survival. J Clin Oncol 2013;31:876-885.
21 Schmid D, Leitzmann MF: Association between physical activity and mortality among breast cancer and colorectal cancer survivors: a systematic review and meta-analysis. Ann Oncol 2014;25:1293-1311.

22 Courneya KS, Friedenreich CM, Quinney HA, Fields AL, Jones LW, Fairey AS: A randomized trial of exercise and quality of life in colorectal cancer survivors. Eur J Cancer Care (Engl) 2003;12:347-357.

23 Pisani P: Hyper-insulinaemia and cancer, meta-analyses of epidemiological studies. Arch Physiol Biochem 2008;114:63-70.

24 Inoue M, Tsugane S: Insulin resistance and cancer: epidemiological evidence. Endocr Relat Cancer 2012;19: F1-8.

25 Demarzo MM, Martins LV, Fernandes CR, Herrero FA, Perez SE, Turatti A, Garcia SB: Exercise reduces inflammation and cell proliferation in rat colon carcinogenesis. Med Sci Sports Exerc 2008;40:618-621.

26 Rigas B, Tsioulias GJ: The evolving role of nonsteroidal anti-inflammatory drugs in colon cancer prevention: a cause for optimism. J Pharmacol Exp Ther 2015;353: $2-8$.

27 Yamauchi M, Lochhead P, Imamura Y, et al: Physical activity, tumor PTGS2 expression, and survival in patients with colorectal cancer. Cancer Epidemiol Biomarkers Prev 2013;22:1142-1152.

28 Aoi W, Naito Y, Takagi T, et al: A novel myokine, secreted protein acidic and rich in cysteine (SPARC), suppresses colon tumorigenesis via regular exercise. Gut 2013;62:882-889.

29 Wolin KY, Yan Y, Colditz GA: Physical activity and risk of colon adenoma: a meta-analysis. Br J Cancer 2011;104:882-885.

30 Meyerhardt JA, Niedzwiecki D, Hollis D, et al: Impact of body mass index and weight change after treatment on cancer recurrence and survival in patients with stage III colon cancer: findings from Cancer and Leukemia Group B 89803. J Clin Oncol 2008;26:4109-4115.

31 Campbell PT, Newton CC, Dehal AN, Jacobs EJ, Patel $\mathrm{AV}$, Gapstur SM: Impact of body mass index on survival after colorectal cancer diagnosis: The Cancer Prevention Study-II Nutrition Cohort. J Clin Oncol 2012; 30:42-52.
32 Meyerhardt JA, Niedzwiecki D, Hollis D, Saltz LB, Hu FB, Mayer RJ, Nelson H, Whittom R, Hantel A, Thomas J, Fuchs CS: Association of dietary patterns with cancer recurrence and survival in patients with stage III colon cancer. JAMA 2007;298:754-764.

$33 \mathrm{Ng} \mathrm{K}$, Meyerhardt JA, Wu K, Feskanich D, Hollis BW, Giovannucci EL, Fuchs CS: Circulating 25-hydroxyvitamin D levels and survival in patients with colorectal cancer. J Clin Oncol 2008;26:2984-2991.

34 Cole BF, Baron JA, Sandler RS, et al: Folic acid for the prevention of colorectal adenomas: a randomized clinical trial. JAMA 2007;297:2351-2359.

35 Gravitz L: Prevention: tending the gut. Nature 2015; 521:S6-8.

36 Bockelman C, Engelmann BE, Kaprio T, Hansen TF, Glimelius B: Risk of recurrence in patients with colon cancer stage II and III: a systematic review and metaanalysis of recent literature. Acta Oncol 2015;54:5-16.

37 Brenner H, Kloor M, Pox CP: Colorectal cancer. Lancet 2014;383:1490-1502.

38 Martling AL, Holm T, Rutqvist LE, Moran BJ, Heald RJ, Cedemark B: Effect of a surgical training programme on outcome of rectal cancer in the County of Stockholm. Stockholm Colorectal Cancer Study Group, Basingstoke Bowel Cancer Research Project. Lancet 2000;356:93-96.

39 Gaedcke J, Liersch T, Hess C, Becker H, Rodel C, Ghadimi BM: Rectal cancer: current status of multimodal therapy - when and how? (Article in German). Zentralbl Chir 2011;136:334-342.

40 Orlich MJ, Singh PN, Sabate J, et al: Vegetarian dietary patterns and the risk of colorectal cancers. JAMA Intern Med 2015;175:767-776.

41 World Cancer Research Fund/American Institute for Cancer Research: Food, Nutrition, Physical Activity, and the Prevention of Cancer: A Global Perspective. Washington, DC, AICR/WCRF, 2007. www.aicr.org/ assets/docs/pdf/reports/Second_Expert_Report.pdf. 